\title{
Occurrence and Multilocus Genotyping of Giardia duodenalis in Yunnan Black Goats in China
}

\author{
Shi-Chen Xie $\mathbb{D}^{1,},{ }^{1,2}$ Yang Zou, ${ }^{2}$ Dan Chen, ${ }^{2}$ Meng-Meng Jiang, ${ }^{1}$ Xiao-Dan Yuan, ${ }^{2}$ Zhao Li, ${ }^{3}$ \\ Feng-Cai Zou, ${ }^{3}$ Jian-Fa Yang, ${ }^{3}$ Jin-Liang Sheng $\left(\mathbb{D},{ }^{1}\right.$ and Xing-Quan Zhu $\mathbb{D}^{2,4}$ \\ ${ }^{1}$ College of Animal Science and Technology, Shihezi University, Shihezi, Xinjiang Uygur Autonomous Region 832003, China \\ ${ }^{2}$ State Key Laboratory of Veterinary Etiological Biology, Key Laboratory of Veterinary Parasitology of Gansu Province, \\ Lanzhou Veterinary Research Institute, Chinese Academy of Agricultural Sciences, Lanzhou, Gansu Province 730046, China \\ ${ }^{3}$ Key Laboratory of Veterinary Public Health of Yunnan Province, College of Veterinary Medicine, Yunnan Agricultural University, \\ Kunming, Yunnan Province 650201, China \\ ${ }^{4}$ Jiangsu Co-innovation Center for the Prevention and Control of Important Animal Infectious Diseases and Zoonoses, \\ Yangzhou University College of Veterinary Medicine, Yangzhou, Jiangsu Province 225009, China
}

Correspondence should be addressed to Jin-Liang Sheng; sjlshz@126.com and Xing-Quan Zhu; zhuxingquan@caas.cn

Received 6 August 2018; Revised 15 September 2018; Accepted 26 September 2018; Published 10 October 2018

Academic Editor: Roberto Amerigo Papini

Copyright ( $\odot 2018$ Shi-Chen Xie et al. This is an open access article distributed under the Creative Commons Attribution License, which permits unrestricted use, distribution, and reproduction in any medium, provided the original work is properly cited.

Giardia duodenalis is an important zoonotic parasite which can parasitize in the intestines of humans and various animals. However, the information about the prevalence and genetic diversity of G. duodenalis in goats in China is limited. It is yet to be known whether Yunnan black goats, a unique goat breed in subtropical Yunnan province, southwestern China, are infected with G. duodenalis. Thus, a total of $907 \mathrm{fecal}$ samples were collected from Yunnan black goats in five regions in Yunnan province, to estimate the prevalence and genotypes of G. duodenalis using a PCR-based approach. The G. duodenalis prevalence is 4.2\% (38/907) in Yunnan black goats by nested amplification of the $\beta$-giardin (bg) gene, and the genotypes are identified as assemblage $\mathrm{E}$, with 5 novel subtypes ( $\mathrm{E}_{11}$ $\mathrm{E}_{15}$ ). Multilocus sequence typing revealed that 11,18 , and 38 samples were amplifiable on tpi (triose phosphate isomerase), gdh (glutamate dehydrogenase), and bg locus, respectively, and identified three novel multilocus genotypes (MLGs): MLGE9-MLGE11. To our knowledge, this is the first report of G. duodenalis prevalence and genotypes in Yunnan black goats in China, which extended the host range of $G$. duodenalis and provided basic data for controlling G. duodenalis infection in Yunnan black goats.

\section{Introduction}

Giardia duodenalis (syn. Giardia lamblia and Giardia intestinalis) is a common enteric protozoan parasite which can infect humans and a wide range of animal species. $G$. duodenalis infection can cause a series of diseases which have important effects on human and animal health, such as abdominal cramps, diarrhea, weight loss, and malabsorption [1-3]. G. duodenalis infection can be caused by ingesting cysts in contaminated water or food, or through fecal-oral access due to wastewater [4-6]. According to the existing literature, the prevalence of $G$. duodenalis is approximately $10 \%$ in the world population [7], and the G. duodenalis prevalence ranged from 0 to $15.6 \%$ in humans $[8,9]$ and $1.3 \%$ $55.6 \%$ in sheep and goats in China [1]. G. duodenalis has a high prevalence in some low-income areas and developing countries [10-13].

So far, G. duodenalis isolates from humans and various animals have been classified into eight different assemblages $(\mathrm{A}-\mathrm{H})$ on the basis of molecular characterization $[14,15]$. Among them, assemblages $\mathrm{A}$ and $\mathrm{B}$ are the important zoonotic parasites that have a wild range of hosts, including human and other mammals, such as bovine, sheep, goats, and other domestic animals [1, 3]. Assemblage E occurs in artiodactyls, and assemblages C, D, F, G, and $\mathrm{H}$ have obvious animal specificity, but assemblages $\mathrm{C}-\mathrm{F}$ have also been reported in humans in Ethiopia [16], Thailand [17], and Egypt [18].

Yunnan province is the fifth largest producer of goats in China [19], and about 10 million goats are raised each year. 
Many previous studies have reported $G$. duodenalis infection in goats in other countries with prevalence ranging from 2.9 to $35.8 \%[20,21]$, but only limited investigations have been conducted in goats in China, with the prevalence ranging from 2.9 to $7.1 \%$ [22-25].

Yunnan black goat is a unique breed of goat distributed in subtropical Yunnan province, southwestern China. It is yet to be known whether Yunnan black goats are infected with G. duodenalis. Thus, the objectives of the present study were to estimate the $G$. duodenalis prevalence in Yunnan black goats based on characterization of the $\beta$-giardin $(b g)$ gene sequences and identify its genotypes using multilocus genotyping (MLG) targeting gdh gene, tpi gene, and bg gene sequences $[15,26]$.

\section{Materials and Methods}

2.1. Animals and Samples Collection. A total of 907 fecal samples were randomly collected from Yunnan black goats in Chuxiong, Lijiang, and Xishuangbanna prefectures, Yunnan province, southwestern China (Figure 1). All of the fecal samples were stored in separate sterile plastic collection tubes containing $2.5 \%$ potassium dichromate, kept cold with ice packs, transported to the laboratory as soon as possible, and kept in $4^{\circ} \mathrm{C}$ freezer until analysis. The sample information including geographical gender, age, locality, and date of sampling was recorded.

2.2. Genomic DNA Extraction. Fecal specimens were washed repeatedly with ultrapure water until all the potassium dichromate was removed, and then genomic DNA was extracted from $200 \mathrm{mg}$ of each fecal sample in a $2 \mathrm{ml}$ centrifuge tube using the commercial E.Z.N.A ${ }^{\circledR}$ Stool DNA kit (Omega Bio-Tek Inc., GA, USA) by following the manufacturer's instruction. The obtained DNA samples were stored at $-20^{\circ} \mathrm{C}$ for further study.

2.3. PCR Amplification and Sequencing. Each fecal specimen was examined for the presence and genotype of $G$. duodenalis by PCR-based sequencing of the $511 \mathrm{bp}$ fragment of the bggene [27]. In addition, For MLG analysis, all bg-positive specimens were subjected to further PCR using primers for the tpi gene loci and gdh gene loci [28-30]. The sequences of primers are presented in Table 1.

The secondary reaction mixture contained $2 \mu \mathrm{l}$ of template from the first PCR product, $2 \mu \mathrm{L}$ deoxyribonucleotide triphosphate (dNTP) mixture, $2.5 \mu \mathrm{L}$ of $10 \times \mathrm{PCR}$ buffer, $3 \mathrm{mM}$ of $\mathrm{MgCl}_{2}$, and $0.2 \mu \mathrm{M}$ of each primer in a total volume of $25 \mu \mathrm{L}$. PCR amplifications were performed as follows: 1 cycle for $5 \mathrm{~min}$ at $94^{\circ} \mathrm{C}$, followed by 35 cycles of $45 \mathrm{~s}$ at $94^{\circ} \mathrm{C}$ for denaturation, $45 \mathrm{~s}$ at $67^{\circ} \mathrm{C}$ for annealing, and $45 \mathrm{~s}$ at $72^{\circ} \mathrm{C}$ for an extension. All of amplification products were subsequently visualized on $1.5 \%$ agarose gels with ethidium bromide. For each PCR amplification, a positive sample (sequenced DNA) and negative (PCR water) control sample were included.

All nested-PCR products were sent to Xian Qingk Biotechnology Company for two-directional sequencing on an ABI PRISM 3730 XL DNA Analyzer (Applied Biosystems, Foster City, CA, USA) using relevant internal nested primers for PCR amplification. The sequences obtained were compared with relevant sequences available in GenBank database (http://www.ncbi.nlm.nih.gov/GenBank) using Basic Local Alignment Search Tool (BLAST).

2.4. Phylogenetic Analysis. The tpi gene sequences were used for phylogenetic reconstruction using the Neighbor-Joining [NJ] analysis and the genetic distances were calculated by the Kimura 2-parameter model in MEGA6 [31, 32]. Bootstrap analysis (1000 replicates) was used to evaluate the reliability of the phylogenetic tree [33].

2.5. Statistical Analysis. The relationships between G. duodenalis prevalence and risk factors were analyzed using the $x^{2}$ test in SPSS 20.0 (SPSS Inc., Chicago, IL, USA), and statistically significant differences were considered when $P$ $<0.05$.

\section{Results and Discussion}

3.1. The Prevalence of G. duodenalis in Yunnan Black Goats. A total of 907 fecal samples were collected from Yunnan black goats in five regions in Yunnan province (Figure 1), and 38 (4.2\%, 95\% CI, 2.9-5.5) were G. duodenalis-positive based on the amplification of the bg gene. G. duodenalis prevalence was significantly different among the study areas $(\chi 2=10.933$, $d f=4, P<0.05)$, between different age groups $(\chi 2=5.208$, $d f=1, P<0.05)$, and between different genders $(\chi 2=1.615$, $d f=1, P>0.05)$. The $G$. duodenalis prevalence in Yunnan black goats was higher than that $(2.9 \%)$ in goats in Heilongjiang province [22], but lower than that in goats in Anhui (6.3\%) [23], Shaanxi (7.9\%) [25], and Henan provinces $(12.7 \%)$ [24], China. The G. duodenalis prevalence in Yunnan black goats was markedly lower than in goats in Greece $(40.4 \%)$ [34], Spain (42.0\%) [20], Uganda (40.7\%) [35], and Belgium (35.8\%) [21]. The difference in G. duodenalis prevalence may be related to feeding conditions, geographical difference, and animal husbandry practices as well as different susceptibility of different breeds of goats.

G. duodenalis prevalence ranged from $0 \%$ to $7.03 \%$ among the sampled areas. The highest $G$. duodenalis prevalence was found in Yunnan black goats in Mohan (7.03\%, 9/128), Xishuangbanna prefecture (Table 2), followed by Wuding $(5.41 \%, 24 / 444)$ in Chuxiong prefecture, Ninglang $(1.96 \%$, $1 / 51)$ in Lijiang prefecture, and Yongreng (1.43\%, 2/139) and Mouding $(1.38 \%, 2 / 145)$ in Chuxiong prefecture. The likely reason for this discrepancy may be due to different geographical conditions.

3.2. Molecular Characterization of G. duodenalis Isolates. All the bg sequences obtained in the present study were aligned with corresponding $G$. duodenalis sequences available in GenBank by BLAST. A total of 38 positive samples were clustered in assemblage $\mathrm{E}$, containing one known assemblage E subtype $\left(E_{5}, n=35\right)$ and two novel assemblage E subtypes (designated as $\mathrm{E}_{14}, \mathrm{n}=1 ; \mathrm{E}_{15}, \mathrm{n}=2$ ) based on sequence analyses of the bg gene loci (Table 3). Additionally, one known assemblage $\mathrm{E}$ subtype $\left(\mathrm{E}_{10}, \mathrm{n}=2\right)$ and one novel assemblage E subtype $\left(E_{13}, n=16\right)$ based on the gdh gene sequences and 
TABLE 1: Primers used in the study; annealing temperatures used in the PCRs.

\begin{tabular}{|c|c|c|c|c|}
\hline Gene & Primer & Sequences $\left(5^{\prime}-3^{\prime}\right)$ & Annealing temperature $\left({ }^{\circ} \mathrm{C}\right)$ & Reference \\
\hline \multirow[t]{4}{*}{ bg } & GF1 & AAGCCCGACGACCTCACCCGCAGTGC & 55 & [1] \\
\hline & GR1 & GAGGCCGCCCTGGATCTTCGAGACGAC & & \\
\hline & GF2 & GAACGAACGAGATCGAGGTCCG & 55 & \\
\hline & GR2 & CTCGACGAGCTTCGTGTT & & \\
\hline \multirow[t]{4}{*}{ gdh } & Gdhl & TTCCGTRTYCAGTACAACTC & 50 & [1] \\
\hline & Gdh2 & ACCTCGTTCTGRGTGGCGCA & & \\
\hline & Gdh3 & ATGACYGAGCTYCAGAGGCACGT & 65 & \\
\hline & Gdh4 & GTGGCGCARGGCATGATGCA & & \\
\hline \multirow[t]{4}{*}{ tpi } & AL3543 & AAATIATGCCTGCTCGTCG & 50 & {$[1,3]$} \\
\hline & AL3546 & CAA ACCTTITCCGCAAACC & & \\
\hline & ALEf & CCCCTTCTGCCGTACATTTAT & 58 & \\
\hline & ALEr & GGCTCGTAAGCAATAACGACTT & & \\
\hline
\end{tabular}

TABLE 2: Prevalence and risk factors of Giardia duodenalis infection in Yunnan black goats in Yunnan province, southwestern China.

\begin{tabular}{|c|c|c|c|c|c|}
\hline Factor & Category & No. tested & $\begin{array}{c}\text { No. positive (\%) } \\
{[95 \% \mathrm{CI}]}\end{array}$ & OR [95 \% CI] & $P$-value \\
\hline \multirow[t]{5}{*}{ Area } & Wuding & 444 & $24(5.4,3.3-7.5)$ & $4.086(0.95-17.50)$ & 0.04 \\
\hline & Yongreng & 139 & $2(1.4,0.6-3.3)$ & $1.044(0.15-7.51)$ & 0.97 \\
\hline & Mouding & 145 & $2(1.4,0.5-3.3)$ & Ref & Ref \\
\hline & Ninglang & 51 & $1(2.0,1.8-5.8)$ & $1.430(0.13-16.11)$ & 0.77 \\
\hline & Mohan & 128 & $9(7.0,2.6-11.5)$ & $5.408(1.15-25.51)$ & 0.02 \\
\hline \multirow[t]{2}{*}{ Gender } & Female & 633 & $23(3.6,2.1-5.1)$ & \multirow{2}{*}{$0.651(0.33-1.27)$} & \multirow{2}{*}{0.20} \\
\hline & Male & 274 & $15(5.5,2.8-8.2)$ & & \\
\hline \multirow{2}{*}{$\begin{array}{l}\text { Age } \\
\text { (month) }\end{array}$} & $\leq 12$ & 364 & $22(6.1,3.6-8.6)$ & \multirow{3}{*}{$2.119(1.10-4.09)$} & \multirow{2}{*}{0.02} \\
\hline & $>12$ & 543 & $16(2.9,1.2-4.6)$ & & \\
\hline Total & & 907 & $38(4.2,2.9-5.5)$ & & \\
\hline
\end{tabular}

TABLE 3: Intra-assemblage substitutions in tpi, gdh, and bg loci within Giardia duodenalis assemblage E.

\begin{tabular}{|c|c|c|c|c|c|c|}
\hline \multirow{2}{*}{$\begin{array}{l}\text { Subtypes (number) } \\
\text { tpi }\end{array}$} & \multicolumn{5}{|c|}{ Nucleotide position and substitutions } & \multirow{2}{*}{ GenBank ID } \\
\hline & & & & & & \\
\hline & 188 & 248 & & & & \\
\hline Ref. sequence & G & A & & & & MF095054 \\
\hline $\mathrm{E}_{11}(1)$ & $\mathrm{C}$ & $\mathrm{T}$ & & & & MH621338 \\
\hline \multirow{3}{*}{$\begin{array}{l}\mathrm{E}_{12}(10) \\
\operatorname{gdh}\end{array}$} & G & $\mathrm{A}$ & & & & MH621340 \\
\hline & & & & & & \\
\hline & 391 & 608 & 623 & & & \\
\hline Ref. sequence & $\mathrm{C}$ & A & A & & & KX813711 \\
\hline $\mathrm{E}_{10}(2)$ & $\mathrm{T}$ & G & G & & & \\
\hline \multirow{2}{*}{$\begin{array}{l}E_{13}(16) \\
\text { bg }\end{array}$} & $\mathrm{C}$ & G & G & & & MH621339 \\
\hline & 62 & 66 & 78 & 82 & 365 & \\
\hline Ref. sequence & $\mathrm{C}$ & A & A & $\mathrm{T}$ & $\mathrm{C}$ & KY769092 \\
\hline $\mathrm{E}_{5}(35)$ & $\mathrm{C}$ & A & A & $\mathrm{T}$ & $\mathrm{C}$ & \\
\hline $\mathrm{E}_{14}(1)$ & $\mathrm{A}$ & - & G & G & $\mathrm{C}$ & MH621337 \\
\hline $\mathrm{E}_{15}(2)$ & $\mathrm{C}$ & A & A & $\mathrm{T}$ & $\mathrm{T}$ & MH621341 \\
\hline
\end{tabular}


TABLE 4: Multilocus characterization of Giardia duodenalis isolates based on the tpi, gdh, and bg genes.

\begin{tabular}{|c|c|c|c|c|}
\hline subtype & & & No. of sequences & MLG type \\
\hline tpi & gdh & bg & & \\
\hline $\mathrm{E}_{12}$ & $\mathrm{E}_{13}$ & $\mathrm{E}_{15}$ & 1 & MLGE9 \\
\hline $\mathrm{E}_{12}$ & $\mathrm{E}_{13}$ & $\mathrm{E}_{5}{ }^{\mathrm{a}}$ & 8 & MLGE10 \\
\hline $\mathrm{E}_{11}$ & $\mathrm{E}_{13}$ & $\mathrm{E}_{5}{ }^{\mathrm{a}}$ & 1 & MLGE11 \\
\hline- & $\mathrm{E}_{13}$ & $\mathrm{E}_{15}$ & 1 & \\
\hline- & $\mathrm{E}_{10}^{\mathrm{b}}$ & $\mathrm{E}_{5}{ }^{\mathrm{a}}$ & 2 & \\
\hline- & $\mathrm{E}_{13}$ & $\mathrm{E}_{5}{ }^{\mathrm{a}}$ & 5 & \\
\hline $\mathrm{E}_{12}$ & - & $\mathrm{E}_{5}{ }^{\mathrm{a}}$ & 1 & \\
\hline- & - & $\mathrm{E}_{14}$ & 1 & \\
\hline- & - & $\mathrm{E}_{5}{ }^{\mathrm{a}}$ & 18 & \\
\hline
\end{tabular}

Note: $\mathrm{a}, \mathrm{b}$ indicate that genotypes have been reported.

-: not determined.
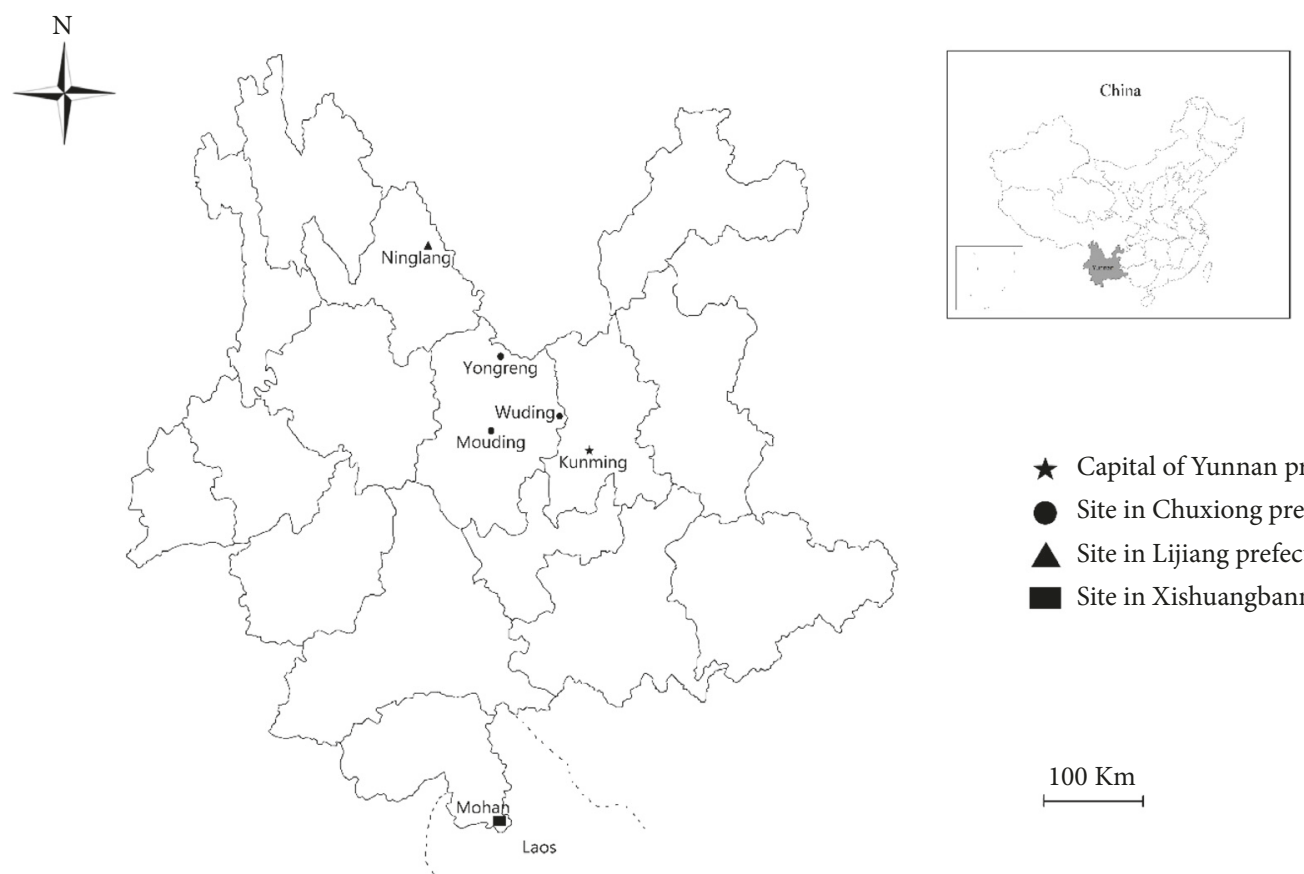

$\star$ Capital of Yunnan province

Site in Chuxiong prefecture

A Site in Lijiang prefecture

Site in Xishuangbanna prefecture

$100 \mathrm{Km}$

FIGURE 1: A map depicting the sampling sites for collecting fecal samples from Yunnan black goats in Yunnan province, southwestern China.

two novel assemblage E subtypes $\left(\mathrm{E}_{11}, \mathrm{n}=1 ; \mathrm{E}_{12}, \mathrm{n}=10\right)$ based on the tpi gene sequences were also identified among $G$. duodenalis-positive samples from Yunnan black goats (Table 3).

Previous studies have indicated that assemblage $\mathrm{E}$ is the predominant genotype infecting a range of hoofed livestock; it is also the most common assemblage found in sheep, goats, and pigs. However, assemblage $\mathrm{E}$ has also been identified in cattle, dogs, cats, horses, fallow deer, monkeys, and humans $[1,3,29,36]$ indicating that assemblage $\mathrm{E}$ is of zoonotic significance.

MLG analysis based on bg, gdh, and tpi gene sequences is a useful tool to illustrate the diversity of the G. duodenalis genotypes [37]. In this study, 18 of the $38 \mathrm{bg}$-positive samples were gdh-positive, and 11 were tpi-positive. Ten samples were successfully sequenced at all of the three loci, and three novel
MLGs (designated as MLGE9-E11) were identified within assemblage E (Table 4).

3.3. Phylogenetic Analysis of $G$. duodenalis Isolates from Yunnan Black Goats. To clarify the genetic relationships of the G. duodenalis isolates in this study with relevant G. duodenalis isolates, the obtained $G$. duodenalis tpi gene sequences were aligned with corresponding sequences available in the GenBank database. The phylogenetic tree showed that $G$. duodenalis isolates from Yunnan black goats clustered within assemblage $\mathrm{E}$ which contained $G$. duodenalis isolates $\left(\mathrm{E}_{11}\right.$ and $E_{12}$ ) from other animals and humans (Figure 2), with a high bootstrap value, indicating that $G$. duodenalis genotypes in Yunnan black goats have zoonotic potential, raising a public health concern. 


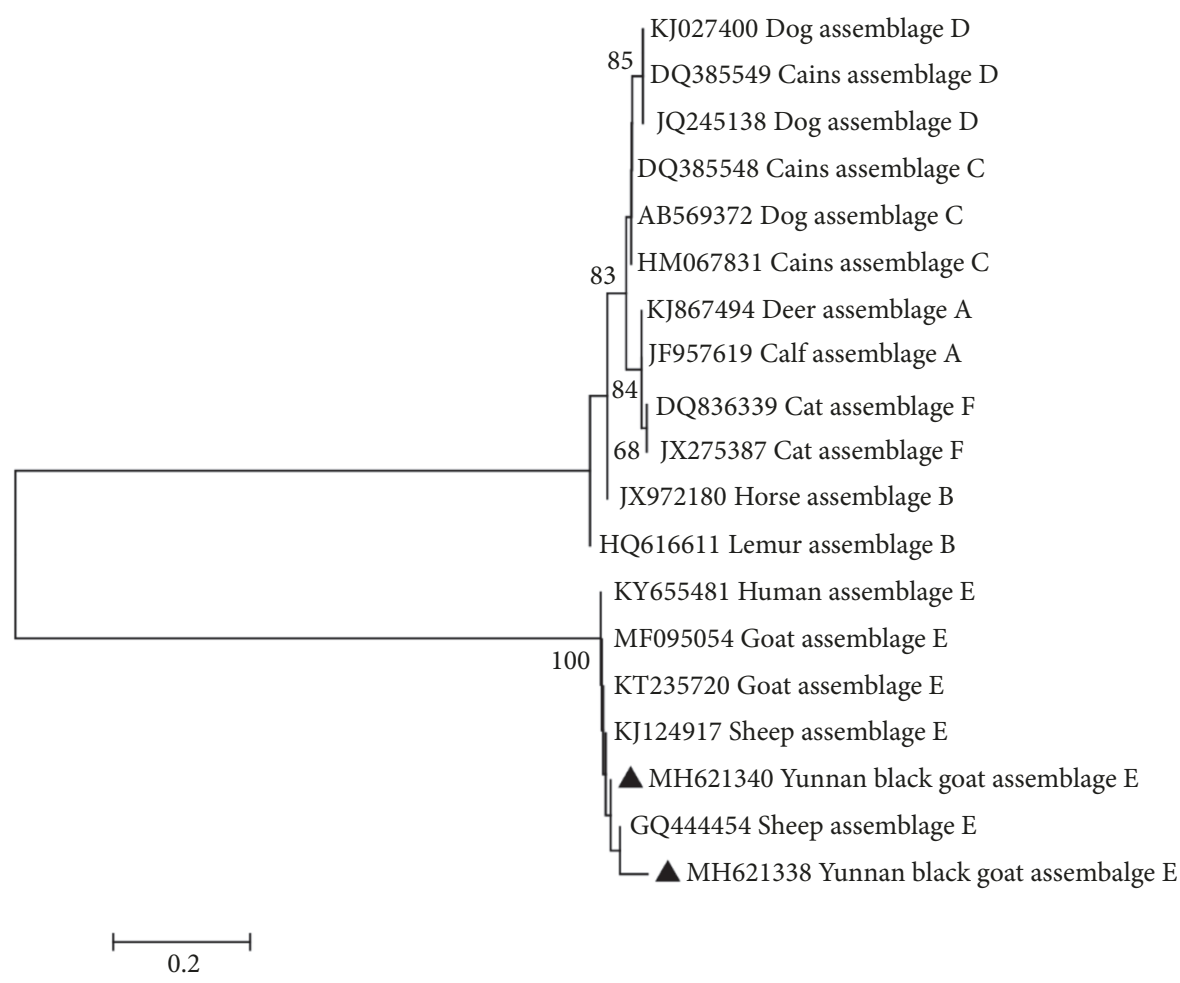

FIgURE 2: The phylogenetic relationships among G. duodenalis isolates inferred by a Neighbor-Joining (NJ) algorithm using a Kimura two-parameter analysis (1000 replicates) based on the tpi gene sequences. The two novel assemblage $\mathrm{E}$ subtypes $\mathrm{E}_{11}$ and $\mathrm{E}_{12}$ (MH621338, MH621340) are marked by filled triangles.

\section{Conclusion}

This is the first report of prevalence and molecular characterization of $G$. duodenalis from Yunnan black goats in Yunnan province, southwestern China, which revealed a $4.2 \%$ G. duodenalis prevalence and identified seven subtypes including five novel assemblages $E$ subtypes $\left(\mathrm{E}_{11}-\mathrm{E}_{15}\right)$ and two known assemblages $\mathrm{E}$ subtypes $\left(\mathrm{E}_{5}\right.$ and $\left.\mathrm{E}_{10}\right)$. MLGs analysis identified three novel MLGs within assemblage $\mathrm{E}$ of G. duodenalis. These results not only extended the host range of $G$. duodenalis distribution, but also enriched the genetic diversity of $G$. duodenalis in humans and animals, which also have implications for controlling $G$. duodenalis infection in Yunnan black goats.

\section{Data Availability}

The Giardia duodenalis prevalence data used to support the findings of this study are included within the article.

\section{Ethical Approval}

All Yunnan black goats were handled in strict accordance with good animal practice according to the Animal Ethics Procedures and Guidelines of the People's Republic of China, and the study was approved by the Animal Administration and Ethics Committee of Lanzhou Veterinary Research Institute, Chinese Academy of Agricultural Sciences.

\section{Conflicts of Interest}

The authors declare that they have no conflicts of interest.

\section{Acknowledgments}

Project support was provided by the Excellent Scientist Fund of Yunnan Agricultural University (2015JY03), the Agricultural Science and Technology Innovation Program (ASTIP) (Grant No. CAAS-ASTIP-2016-LVRI-03), and the Elite Program of Chinese Academy of Agricultural Sciences.

\section{References}

[1] Y. Feng and L. Xiao, "Zoonotic potential and molecular epidemiology of Giardia species and giardiasis," Clinical Microbiology Reviews, vol. 24, no. 1, pp. 110-140, 2011.

[2] W. Li, C. Liu, Y. Yu et al., "Molecular characterization of Giardia duodenalis isolates from police and farm dogs in China," Experimental Parasitology emphasizes, vol. 135, no. 2, pp. 223226, 2013.

[3] U. Ryan and S. M. Cacciò, "Zoonotic potential of Giardia," International Journal for Parasitology, vol. 43, no. 12-13, pp. 943-956, 2013.

[4] C. Kourenti, P. Karanis, and H. Smith, "Waterborne transmission of protozoan parasites: a worldwide review of outbreaks and lessons learnt," Journal of Water and Health, vol. 5, no. 1, pp. $1-38,2007$. 
[5] J. Plutzer, J. Ongerth, and P. Karanis, "Giardia taxonomy, phylogeny and epidemiology: Facts and open questions," International Journal of Hygiene and Environmental Health, vol. 213, no. 5, pp. 321-333, 2010.

[6] A. Efstratiou, J. E. Ongerth, and P. Karanis, "Waterborne transmission of protozoan parasites: Review of worldwide outbreaks - An update 2011-2016," Water Research, vol. 114, pp. 14-22, 2017.

[7] D. B. Huang and A. C. White, "An Updated Review on Cryptosporidium and Giardia," Gastroenterology Clinics of North America, vol. 35, no. 2, pp. 291-314, 2006.

[8] H. Liu, Y. Shen, J. Yin et al., "Prevalence and genetic characterization of Cryptosporidium, Enterocytozoon, Giardia and Cyclospora in diarrheal outpatients in china," BMC Infectious Diseases, vol. 14, no. 1, 2014.

[9] S. Zhang, Y. Zhou, W. Xu et al., "Impact of co-infections with enteric pathogens on children suffering from acute diarrhea in southwest China," Infectious Diseases of Poverty, vol. 5, no. 1, 2016.

[10] J. H. Botero-Garcés, G. M. García-Montoya, D. Grisales-Patiño, D. C. Aguirre-Acevedo, and M. C. Álvarez-Uribe, "Giardia intestinalis and nutritional status in children participating in the complementary nutrition program, Antioquia, Colombia, May to October 2006," Revista do Instituto de Medicina Tropical de São Paulo, vol. 51, no. 3, pp. 155-162, 2009.

[11] J. S. Yoder, J. W. Gargano, R. M. Wallace, M. J. Beach et al., "Giardiasis surveillance-United States, 2009-2010," Morbidity and mortality weekly report. Surveillance Summaries, vol. 61, no. 5, pp. 13-23, 2012.

[12] K. A. Ul Haq, N. A. Gul, H. Muhammad Hammad, Y. Bibi, A. Bibi, and J. Mohsan, "Prevalence of giardia intestinalis and hymenolepis nana in afghan refugee population of mianwali district, pakistan," African Health Sciences, vol. 15, no. 2, pp. 394-400, 2015.

[13] J. E. Painter, J. W. Gargano, S. A. Collier, and J. S. Yoder, "Giardiasis surveillance - United States, 2011-2012," Mmwr Supplements, vol. 64, no. 3, pp. 15-25, 2015.

[14] M. F. Heyworth, "Giardia duodenalis genetic assemblages and hosts," Parasite, vol. 23, article no. 23, 2016.

[15] J. Li, H. Wang, R. Wang, and L. Zhang, "Giardia duodenalis infections in humans and other animals in China," Frontiers in Microbiology, vol. 8, 2017.

[16] T. Gelanew, M. Lalle, A. Hailu, E. Pozio, and S. M. Cacciò, "Molecular characterization of human isolates of Giardia duodenalis from Ethiopia," Acta Tropica, vol. 102, no. 2, pp. 92-99, 2007.

[17] R. J. Traub, T. Inpankaew, S. A. Reid et al., “Transmission cycles of Giardia duodenalis in dogs and humans in Temple communities in Bangkok-A critical evaluation of its prevalence using three diagnostic tests in the field in the absence of a gold standard," Acta Tropica, vol. 111, no. 2, pp. 125-132, 2009.

[18] P. Foronda, M. D. Bargues, N. Abreu-Acosta et al., "Identification of genotypes of Giardia intestinalis of human isolates in Egypt," Parasitology Research, vol. 103, no. 5, pp. 1177-1181, 2008.

[19] National Bureau of Statistics of China, http://www.stats.gov.cn/ english/Statisticaldata/AnnualData.

[20] A. Ruiz, P. Foronda, J. F. González et al., "Occurrence and genotype characterization of Giardia duodenalis in goat kids from the Canary Islands, Spain," Veterinary Parasitology, vol. 154, no. 1-2, pp. 137-141, 2008.
[21] T. Geurden, P. Thomas, S. Casaert, J. Vercruysse, and E. Claerebout, "Prevalence and molecular characterisation of Cryptosporidium and Giardia in lambs and goat kids in Belgium," Veterinary Parasitology, vol. 155, no. 1-2, pp. 142-145, 2008.

[22] W. Zhang, X. Zhang, R. Wang et al., "Genetic characterizations of Giardia duodenalis in sheep and goats in Heilongjiang Province, China and possibility of zoonotic transmission," PLOS Neglected Tropical Diseases, vol. 6, no. 9, Article ID e1826, 2012.

[23] Y. F. Gu, L. K. Wang, Y. Li, L. Li et al., "Prevalence and molecular characterization of Giardia lamblia isolates from goats," Chinese Journal of Parasitology and Parasitic Diseases, vol. 32, no. 5, pp. 401-403, 2014.

[24] X.-Q. Peng, G.-R. Tian, G.-J. Ren et al., "Infection rate of Giardia duodenalis, Cryptosporidium spp. and Enterocytozoon bieneusi in cashmere, dairy and meat goats in China," Infection, Genetics and Evolution, vol. 41, pp. 26-31, 2016.

[25] Y.-L. Yin, H.-J. Zhang, Y.-J. Yuan et al., "Prevalence and multilocus genotyping of Giardia duodenalis from goats in Shaanxi province, northwestern China," Acta Tropica, vol. 182, pp. 202206, 2018.

[26] C. Wielinga, U. Ryan, R. C. Andrew Thompson, and P. Monis, "Multi-locus analysis of Giardia duodenalis intra-Assemblage B substitution patterns in cloned culture isolates suggests subAssemblage $\mathrm{B}$ analyses will require multi-locus genotyping with conserved and variable genes," International Journal for Parasitology, vol. 41, no. 5, pp. 495-503, 2011.

[27] I. M. Sulaiman, R. Fayer, C. Bern et al., "Triosephosphate isomerase gene characterization and potential zoonotic transmission of Giardia duodenalis," Emerging Infectious Diseases, vol. 9, no. 11, pp. 1444-1452, 2003.

[28] C. M. Read, P. T. Monis, and R. C. A. Thompson, "Discrimination of all genotypes of Giardia duodenalis at the glutamate dehydrogenase locus using PCR-RFLP," Infection, Genetics and Evolution, vol. 4, no. 2, pp. 125-130, 2004.

[29] S. M. Caccio and U. Ryan, "Molecular epidemiology of giardiasis," Molecular Biochemical Parasitology, vol. 160, no. 2, pp. 7580, 2008.

[30] T. Geurden, P. Geldhof, B. Levecke et al., "Mixed Giardia duodenalis assemblage A and $\mathrm{E}$ infections in calves," International Journal for Parasitology, vol. 38, no. 2, pp. 259-264, 2008.

[31] K. Tamura, G. Stecher, D. Peterson, A. Filipski, and S. Kumar, "MEGA6: Molecular Evolutionary Genetics Analysis version 6.0," Molecular Biology and Evolution, vol. 30, no. 12, pp. 27252729, 2013.

[32] M. Fantinatti, A. R. Bello, O. Fernandes, and A. M. Da-Cruz, "Identification of Giardia lamblia Assemblage E in humans points to a new anthropozoonotic cycle," The Journal of Infectious Diseases, vol. 214, no. 8, pp. 1256-1259, 2016.

[33] N. Saitou and M. Nei, “The neighbor-joining method: a new method for reconstructing phylogenetic trees," Molecular Biology and Evolution, vol. 4, no. 4, pp. 406-425, 1987.

[34] N. Tzanidakis, S. Sotiraki, E. Claerebout et al., "Occurrence and molecular characterization of Giardia duodenalis and Cryptosporidium spp. in sheep and goats reared under dairy husbandry systems in Greece," Parasite, vol. 21, article 45, 2014.

[35] A. R. Johnston, T. R. Gillespie, I. B. Rwego, T. L. T. McLachlan, A. D. Kent, and T. L. Goldberg, "Molecular epidemiology of crossspecies Giardia duodenalis transmission in Western Uganda," PLOS Neglected Tropical Diseases, vol. 4, no. 5, Article ID e683, 2010.

[36] M. Santin, D. Dargatz, and R. Fayer, "Prevalence of Giardia duodenalis assemblages in weaned cattle on cow-calf operations 
in the United States," Veterinary Parasitology, vol. 183, no. 3-4, pp. 231-236, 2012.

[37] S. M. Cacciò, R. Beck, M. Lalle, A. Marinculic, and E. Pozio, "Multilocus genotyping of Giardia duodenalis reveals striking differences between assemblages A and B," International Journal for Parasitology, vol. 38, no. 13, pp. 1523-1531, 2008. 


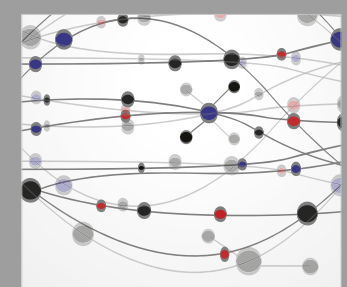

The Scientific World Journal
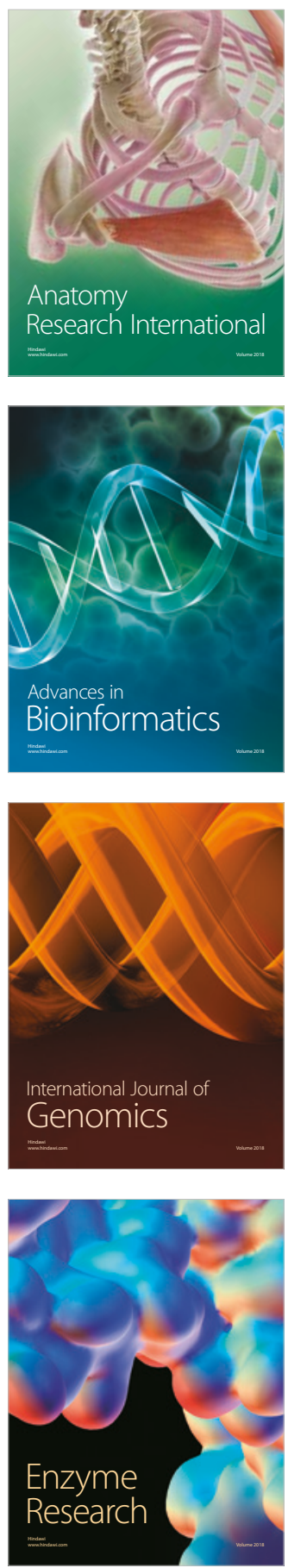
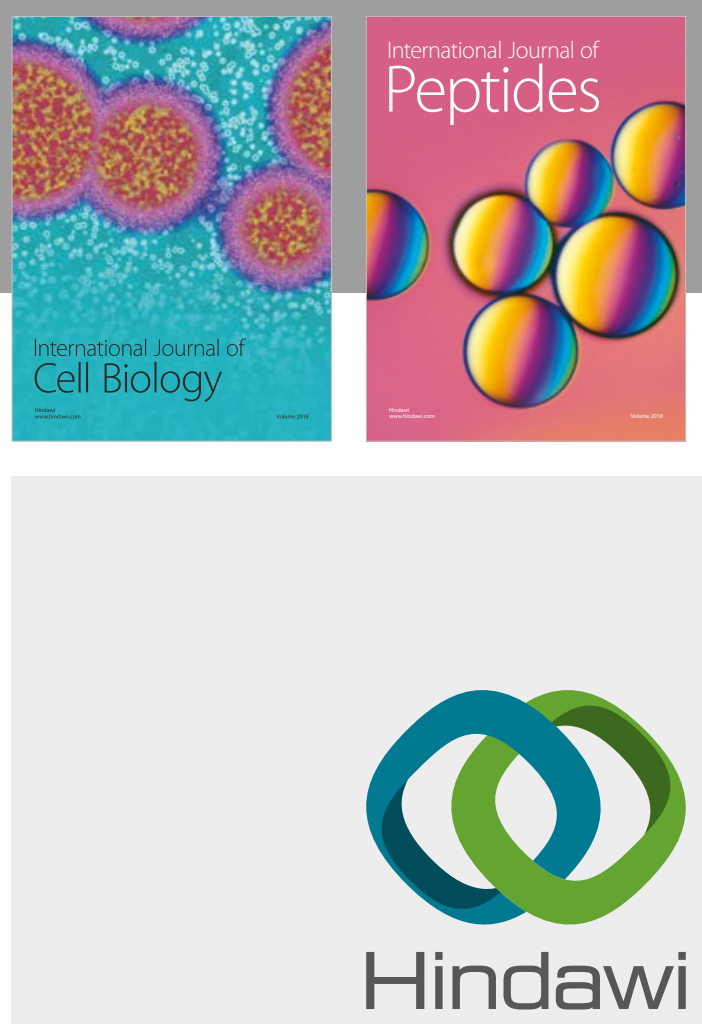

Submit your manuscripts at

www.hindawi.com
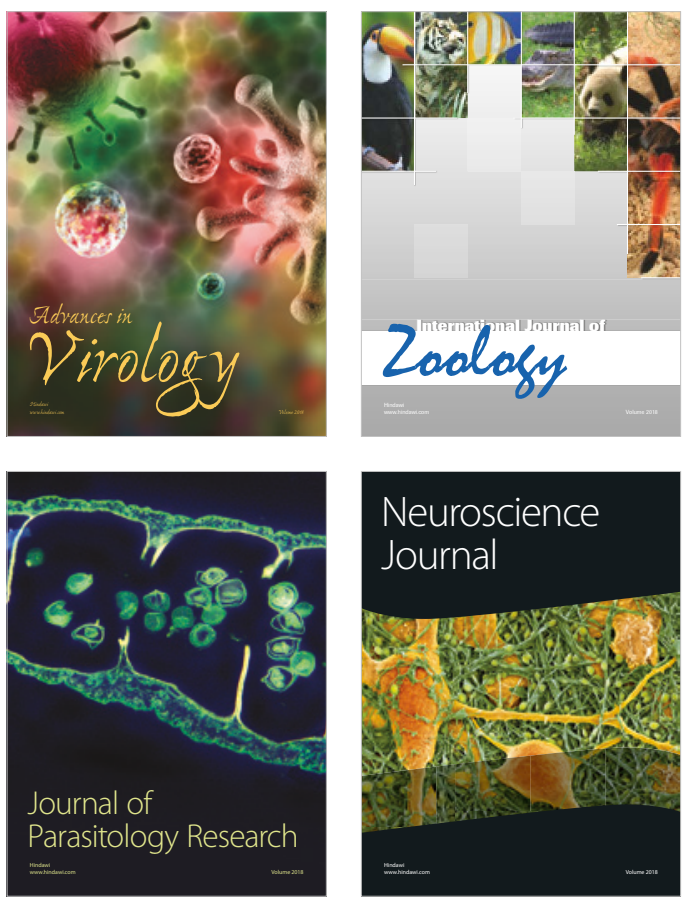
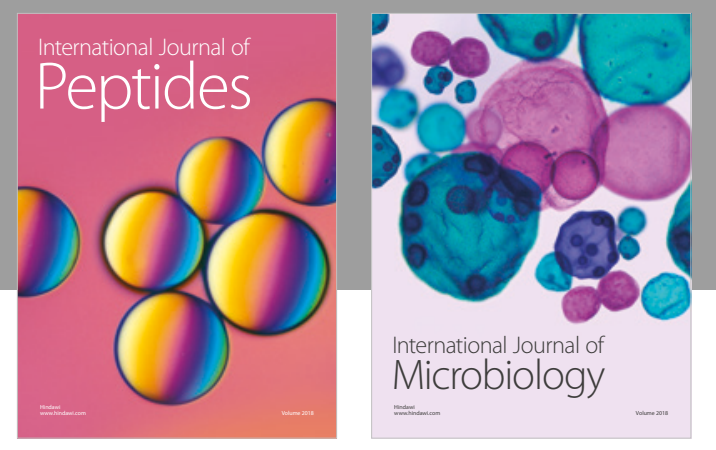

nternational Journal of Microbiology
Journal of
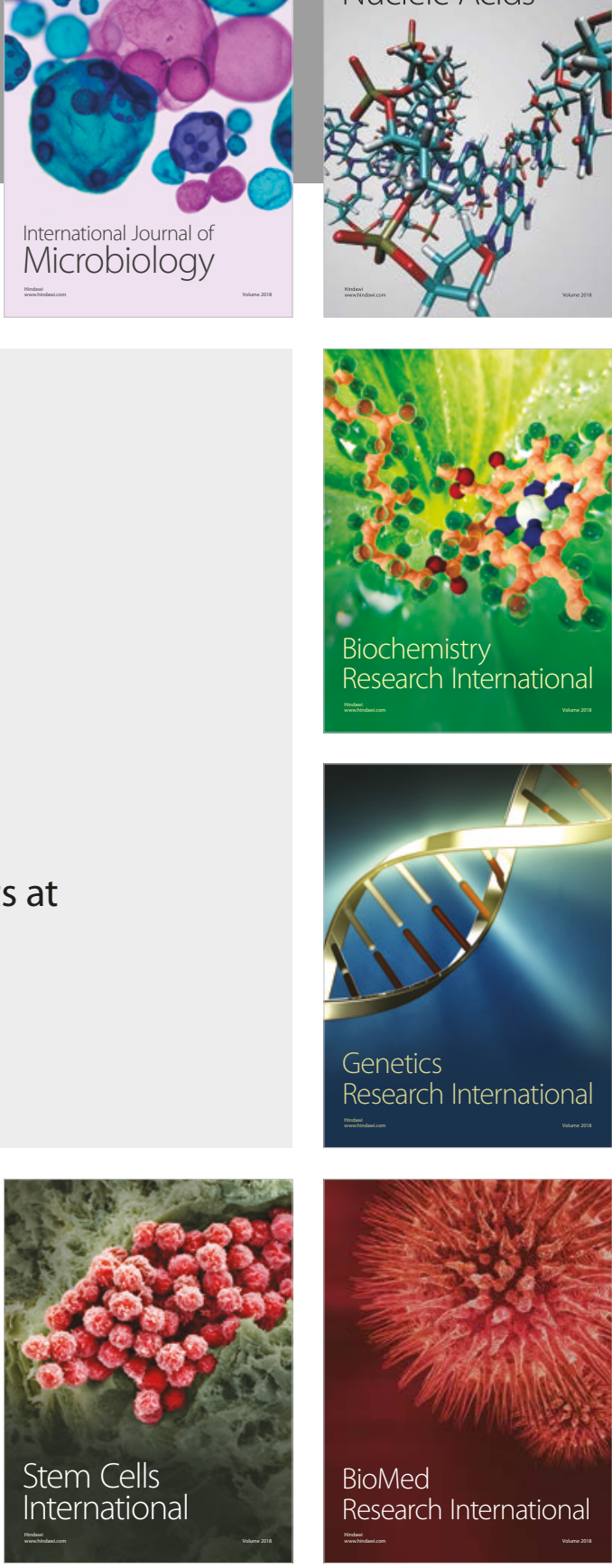
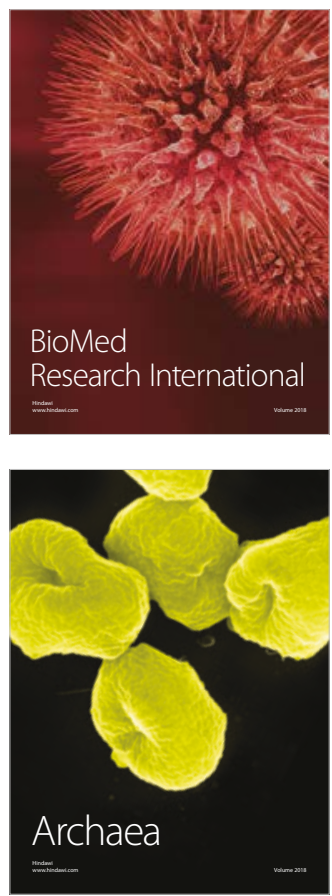